\title{
Prevalence of Pulmonary Embolism in COVID-19: a Pooled Analysis
}

\author{
Rupak Desai ${ }^{1}$ (D) Zainab Gandhi ${ }^{2} \cdot$ Sandeep Singh ${ }^{3} \cdot$ Sonali Sachdeva $^{4} \cdot$ Pritika Manaktala $^{5} \cdot$ Sejal Savani $^{6}$. \\ Virmitra Desai $^{7} \cdot$ Rajesh Sachdeva ${ }^{1,8,9} \cdot$ Gautam Kumar $^{1,10}$
}

Accepted: 19 October 2020 / Published online: 28 October 2020

(C) Springer Nature Switzerland AG 2020

\begin{abstract}
There remains a high risk of thrombosis in patients affected by the SARS-CoV-2 virus and recent reports have shown pulmonary embolism (PE) as a cause of sudden death in these patients. However, the pooled rate of this deadly and frequently underdiagnosed condition among COVID-19 patients remains largely unknown. Given the frequency with which pulmonary embolism has been reported as a fatal complication of severe coronavirus disease, we sought to ascertain the actual prevalence of this event in COVID-19 patients. Using PubMed/Medline, EMBASE, and SCOPUS, a thorough literature search was performed to identify the studies reporting rate of PE among COVID-19. Random effects models were obtained to perform a meta-analysis, and $I^{2}$ statistics were used to measure inter-study heterogeneity. Among 3066 COVID-19 patients included from 9 studies, the pooled prevalence of PE was $15.8 \%\left(95 \% \mathrm{CI}(6.0-28.8 \%), I^{2}=98 \%\right)$. The pooled rate in younger cohort (age $<65$ years) showed a higher prevalence of $20.5 \%$ (95\% CI (17.6-24.8\%)) as compared to studies including relatively older cohort (age $>65$ years) showing $14.3 \%$ (95\% CI $(2.9-30.1 \%)$ ) $(p<0.05)$. Single-center studies showed a prevalence of $12.9 \%$ (95\% CI $1.0-30.2 \%)$, while that of multicenter studies was $19.5 \%$ (95\% CI 14.9-25.2\%) $(p<0.05)$. Pulmonary embolism is a common complication of severe coronavirus disease and a high degree of clinical suspicion for its diagnosis should be maintained in critically ill patients.
\end{abstract}

Keywords Pulmonary embolism $\cdot$ Infectious disease $\cdot$ SARS-CoV-2 $\cdot$ COVID-19

\section{Introduction}

COVID-19 is a multisystem disease which increases risk of coagulopathy and thus, venous thromboembolism (VTE) and pulmonary embolism (PE) in critically ill patients. The incidence rate for VTE and arterial thrombosis and related thrombo-embolic complications remain very high in COVID-19 patients [1]. There are three possible mechanisms

This article is part of the Topical Collection on COVID-19

Rupak Desai

rdesa30@emory.edu; drrupakdesai@gmail.com

1 Division of Cardiology, Atlanta VA Medical Center, Decatur, GA 30033, USA

2 Department of Internal Medicine, Geisinger Community Medical Center, Scranton, PA, USA

3 Department of Clinical Epidemiology, Biostatistics and Bioinformatics, Academic Medical Center,

Amsterdam, The Netherlands

4 Department of Medicine, Lady Hardinge Medical College, New Delhi, India associated with this observation: (1) through cytokine storm by interleukin-1, interleukin-6, tumor necrosis factor-alpha and others leading to endothelial activation and widespread thrombosis as seen in pulmonary vessels in acute respiratory distress syndrome; (2) severe acute respiratory syndrome coronavirus 2 (SARS-CoV-2) has shown an affinity for angiotensin-converting enzyme 2 located at alveolar epithelial cells, endothelial cells of extrapulmonary tissues leading to 
thrombosis by microvascular injury and multiorgan failure; and (3) lupus anticoagulant antibodies can lead to thrombosis by the "two hit" thrombosis model [1].

\section{Methods}

After a thorough review of the literature from PubMed, Scopus, and Google Scholar, data were collected from four studies. Random effects models were obtained to perform a meta-analysis, and $I^{2}$ statistics were used to measure interstudy heterogeneity. $I^{2}>75 \%$ was interpreted as substantial heterogeneity and prevalence was further explored using subgroup analysis.

\section{Results}

Among 3066 COVID-19 patients, the pooled prevalence of PE was $15.8 \%$ (95\% CI $(6.0-28.8 \%)$ ) with $I^{2}=98 \%$. The pooled rate in younger cohort (age $<65$ years) showed a higher prevalence of $20.5 \%$ (95\% CI (17.6-24.8\%)) compared to the older cohort (age $>65$ years) showing $14.3 \%$ (95\% CI $(2.9-30.1 \%))(p<0.00001)$ (Fig. 1). Single-center studies showed a prevalence of $12.9 \%$ (95\% CI $1.0-30.2 \%$ ), while that of multicenter studies was $19.5 \%$ (95\% CI 14.9 $25.2 \%)$.

\section{Discussion}

Due to underlying thrombotic milieu that is characteristic of COVID-19, patients suffering from this infection are highly predisposed to pulmonary embolism which is a major cause of mortality associated with this disease. Patients diagnosed with COVID-19 run the risk of incident pulmonary embolism even in the absence of regular risk factors [2]. Stringent movement restrictions applied as a consequence of this pandemic can lead to a delay in seeking medical attention, subsequently leading to progression of low-risk PE cases to high-risk lifeendangering cases of fatal PE [3].

To the best of our knowledge, this is the first pooled estimate of the prevalence of PE in COVID-19 patients with a variation by age (mean age 65 years) and study centers (single vs. multi). Several single-center/multi-center studies along with case reports have shown increased incidence of PE in COVID-19 patients despite being on prophylactic anticoagulant therapy. In our analysis, we thus proved that multicenter studies with a larger and diverse study sample showed an increased prevalence of PE, thus the need for a low threshold of PE suspicion. The results of our pooled analysis also showed a higher prevalence of PE in the younger cohort (age $<65$ years) compared to elderly patients. A study of
137 critically ill patients in Hubei province with median age of sample $57(20-83)$ years showed that nearly $1 / 5$ th patients had an underlying co-morbidity of hypertension, diabetes mellitus, cardiovascular disease, COPD, malignancy, and others which could further increase the risk of thrombotic events [4]. Thus, in addition to older patients who are known to be at high risk of thrombotic events due to comorbidities, young, or middle-aged groups diagnosed with COVID-19 with underlying diseases must also be screened for PE with a high index of suspicion.

A presentation of PE could range from asymptomatic to chest pain, shortness of breath, hemoptysis, syncope, and overlapping with COVID-19 disease. Thus, initial suspicion towards DVT/PE can be gauged from elevated D-dimer levels. With a higher threshold for D-dimer levels of 1.5 $\mu \mathrm{g} / \mathrm{ml}$, the negative predictive value is reported to be $94.7 \%$ [5]. Thus, with elevated D-dimer levels or clinical symptoms, compression ultrasound could be preceded before CT pulmonary angiography since $90 \%$ of pulmonary emboli are known to originate from lower extremity DVT [5, 6]. Cardiac investigations such as ECG or TTE should not be heavily relied upon; clinical suspicion should be the most important factor driving the clinician towards suspecting and investigation for PE in COVID-19 patients [7].

Risk stratification methods such as Caprini, IMPROVE, Padua models, and VELTAS (Venous and Lymphatic Triage and Acuity Scale) are proven to be useful for clinical assessment of VTE and PE in COVID-19 [8, 9]. Prophylactic anticoagulant therapy is recommended with LMWH or unfractionated heparin with or without mechanical compression [10]. However, recently a case report described fatal thromboembolism in a patient who was already on the usual anticoagulation dose and had a normal D-dimer level until the event [11]. This warrants further research on the effective anticoagulation and therapeutic dose to prevent mortality from this life-threatening complication of COVID-19.

With this pooled analysis, we report that nearly 1 in every 7 COVID-19 patients could present with PE, higher odds of being detected in those with a mean age of less than 65 years ( $20.5 \%$ vs. $14.3 \%$ ) compared to relatively younger cohorts (mean age $<65$ years). As expected, the pooled prevalence in single-center studies remain underreported $(12.9 \%$ vs. $19.4 \%$ ) compared to the pooled rate observed in multicenter studies.

\section{Conclusions}

The level of surveillance for PE and threshold for conducting diagnostic tests such as D-dimer levels need to be increased considering fatal outcomes of a PE with COVID-19. Our study gives preliminary data for further large-scale analysis 
a Random effects Pooled Prevalence of Pulmonary Embolism by Subgroups based on Mean age of study cohort

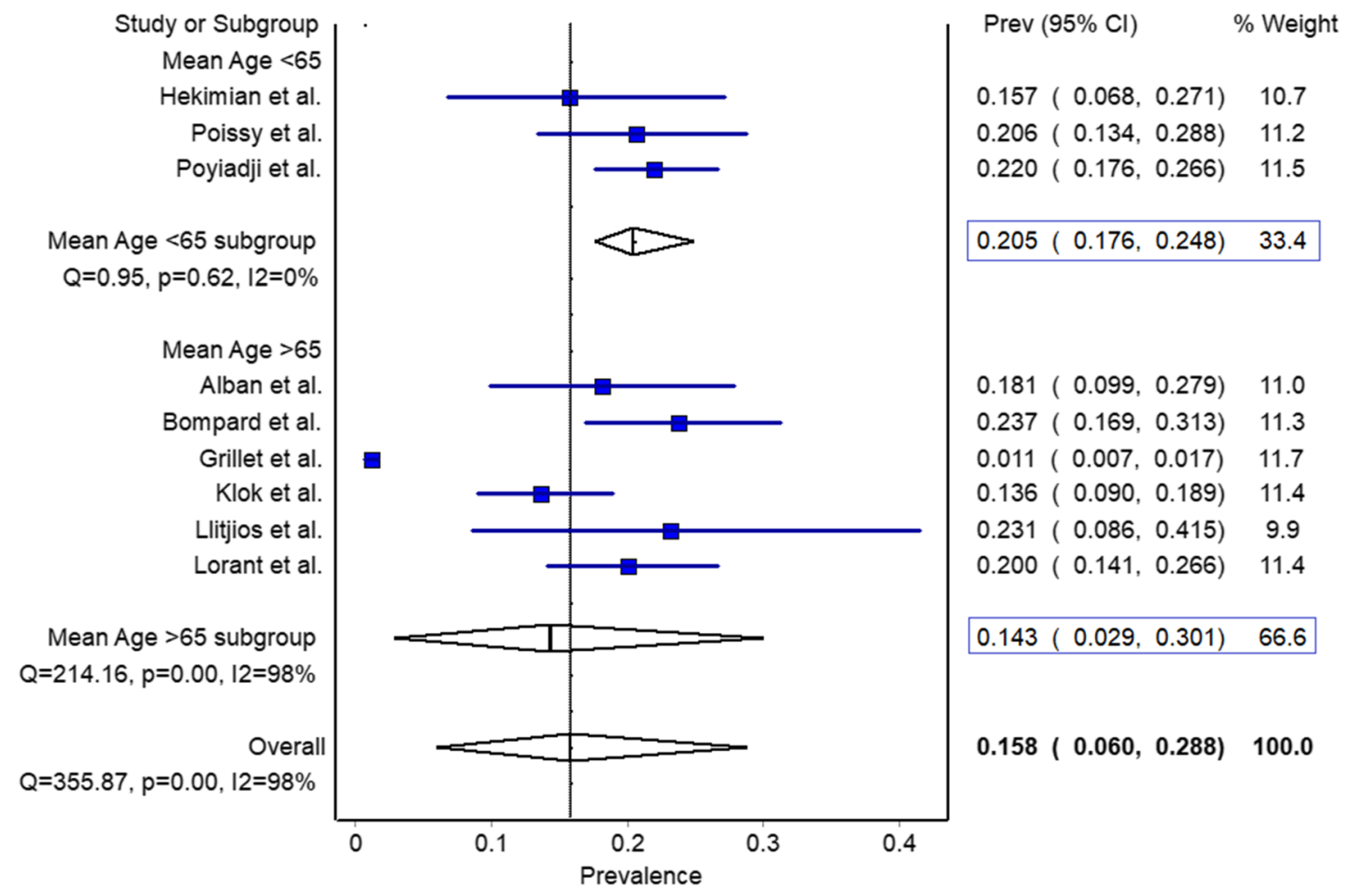

b Random effects Pooled Prevalence of Pulmonary Embolism by Subgroups based on Study centers

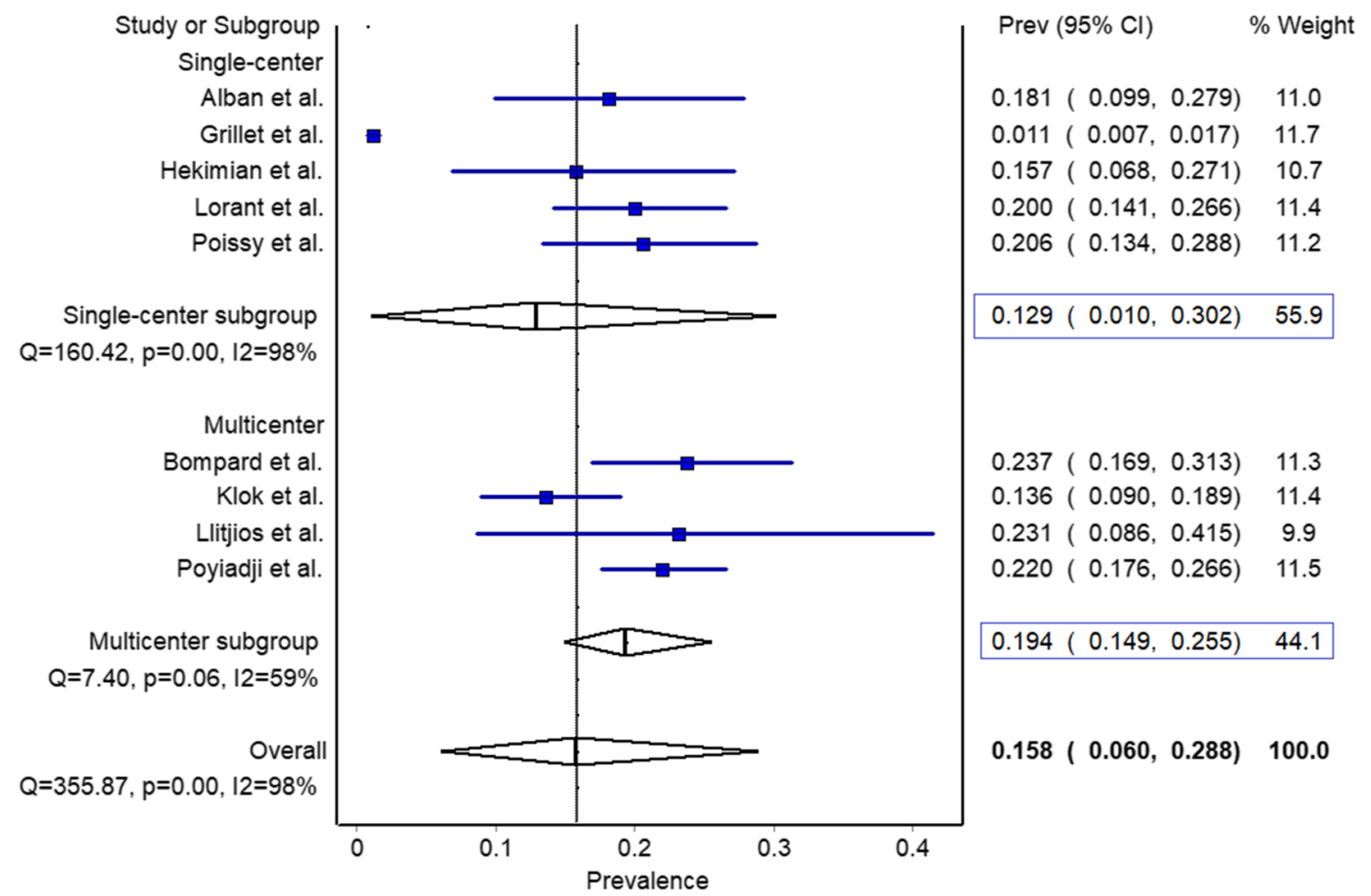

Fig. 1 Pooled prevalence of pulmonary embolism. a The pooled rate in younger cohort (age $<65$ years) showed a higher prevalence of $20.5 \%$ (95\% CI (17.6-24.8\%)) compared to the older cohort (age $>65$ years) showing $14.3 \%(95 \% \mathrm{CI}(2.9-30.1 \%))(p<0.00001)$. b The pooled rate of pulmonary embolism in single-center studies was $12.9 \%$ (95\% CI 1.0 $30.2 \%)$ as compared to $19.4 \%$ (95\% CI $14.9-25.2 \%)$ in multicenter studies $(p<0.00001)$ 
to understand the prevalence of PE in young to middle-aged COVID-19 patients and development of algorithms to speculate and timely diagnose $\mathrm{PE}$ and venous thromboembolism and curtail mortality rate and pertinent complications.

Authors' Contributions Conception and design: Rupak Desai, Sandeep Singh, Zainab Gandhi; literature search: Sonali Sachdeva, Rupak Desai, Sandeep Singh; first draft: Rupak Desai, Sandeep Singh, Sonali Sachdeva, Zainab Gandhi; critical revision and editing: all authors; final approval: all authors.

\section{Compliance with Ethical Standards}

Conflict of Interest The authors declare that they have no conflict of interest.

Ethical Approval This article does not contain any studies with human participants or animals performed by any of the authors.

\section{References}

1. Mucha SR, Dugar S, McCrae K, Joseph D, Bartholomew J, Sacha GL, Militello M. Coagulopathy in COVID-19: Manifestations and management. Cleve Clin J Med. 2020;87(8):461-8. https://doi.org/ 10.3949/ccjm.87a.ccc024.

2. Danzi GB, Loffi M, Galeazzi G, Gherbesi E. Acute pulmonary embolism and COVID-19 pneumonia: a random association? Eur Heart J. 2020;41(19):1858. https://doi.org/10.1093/eurheartj/ ehaa254 PMID: 32227120; PMCID: PMC7184406.

3. Gressenberger P, Gary T, Raggam RB, Borenich A, Brodmann M. Significant increase in the incidence of high-risk pulmonary embolism during the COVID-19 shutdown: the pandemic response causes serious collateral consequences. Eur J Intern Med. 2020: S0953-6205(20)30384-8. https://doi.org/10.1016/j.ejim.2020.10. 007.

4. Liu K, Fang YY, Deng Y, Liu W, Wang MF, Ma JP, et al. Clinical characteristics of novel coronavirus cases in tertiary hospitals in Hubei Province. Chin Med J. 2020;133(9):1025-31. https://doi. org/10.1097/CM9.0000000000000744 PMID: 32044814; PMCID: PMC7147277.
5. Tal S, Spectre G, Kornowski R, Perl L. Venous thromboembolism complicated with COVID-19: what do we know so far? Acta Haematol. 2020;143(5):417-24. https://doi.org/10.1159/ 000508233 PMID: 32396903; PMCID: PMC7270063.

6. Parry AH, Wani AH. Pulmonary embolism in coronavirus disease19 (COVID-19) and use of compression ultrasonography in its optimal management. Thromb Res. 2020;192:36. https://doi.org/ 10.1016/j.thromres.2020.05.022 PMID: 32425262; PMCID: PMC7229911.

7. Kho J, Ioannou A, Van den Abbeele K, Mandal AKJ, Missouris CG. Pulmonary embolism in COVID-19: clinical characteristics and cardiac implications. Am J Emerg Med. 2020:S07356757(20)30649-5. https://doi.org/10.1016/j.ajem.2020.07.054.

8. Boukhris M, Hillani A, Moroni F, Annabi MS, Addad F, Ribeiro $\mathrm{MH}$, et al. Cardiovascular implications of the COVID-19 pandemic: a global perspective. Can J Cardiol. 2020;36(7):1068-80. https://doi.org/10.1016/j.cjca.2020.05.018 PMID: 32425328; PMCID: PMC7229739.

9. Rotzinger DC, Beigelman-Aubry C, von Garnier C, Qanadli SD. Pulmonary embolism in patients with COVID-19: time to change the paradigm of computed tomography. Thromb Res. 2020;190: 58-9. https://doi.org/10.1016/j.thromres.2020.04.011 PMID: 32302782 ; PMCID: PMC7151364.

10. Parsi K, van Rij AM, Meissner MH, Davies AH, De Maeseneer M, Gloviczki $\mathrm{P}$, et al. Triage of patients with venous and lymphatic diseases during the COVID-19 pandemic - the Venous and Lymphatic Triage and Acuity Scale (VELTAS):: a consensus document of the International Union of Phlebology (UIP), Australasian College of Phlebology (ACP), American Vein and Lymphatic Society (AVLS), American Venous Forum (AVF), European College of Phlebology (ECoP), European Venous Forum (EVF), Interventional Radiology Society of Australasia (IRSA), Latin American Venous Forum, Pan-American Society of Phlebology and Lymphology and the Venous Association of India (VAI). J Vasc Surg Venous Lymphat Disord. 2020;8(5):706-10. https:// doi.org/10.1016/j.jvsv.2020.05.002 PMID: 32426220; PMCID: PMC7229740.

11. Abool Maaly CA, Al Suwaidi J, Khatib MY, Alzaeem H. Right atrial thrombus in transient in a COVID-19 patient: clinical echocardiographic features-case report and literature review. SN Compr Clin Med. 2020:1-3. https://doi.org/10.1007/s42399-020-00568-7 PMID: 33047098; PMCID: PMC7542089.

Publisher's Note Springer Nature remains neutral with regard to jurisdictional claims in published maps and institutional affiliations. 